\title{
Identification of bipolar disorder using a combination of multimodality magnetic resonance imaging and machine learning techniques
}

\author{
Hao Li $i^{1,2 \dagger}$, Liqian Cui ${ }^{1,2^{*}+}$, Liping Cao ${ }^{3^{*}}$, Yizhi Zhang ${ }^{3}$, Yueheng Liu ${ }^{4,5}$, Wenhao Deng ${ }^{3}$ and Wenjin Zhou ${ }^{3}$
}

\begin{abstract}
Background: Bipolar disorder (BPD) is a common mood disorder that is often goes misdiagnosed or undiagnosed. Recently, machine learning techniques have been combined with neuroimaging methods to aid in the diagnosis of BPD. However, most studies have focused on the construction of classifiers based on single-modality MRI. Hence, in this study, we aimed to construct a support vector machine (SVM) model using a combination of structural and functional MRI, which could be used to accurately identify patients with BPD.

Methods: In total, 44 patients with BPD and 36 healthy controls were enrolled in the study. Clinical evaluation and MRI scans were performed for each subject. Next, image pre-processing, VBM and ReHo analyses were performed. The ReHo values of each subject in the clusters showing significant differences were extracted. Further, LASSO approach was recruited to screen features. Based on selected features, the SVM model was established, and discriminant analysis was performed.

Results: After using the two-sample t-test with multiple comparisons, a total of 8 clusters were extracted from the data (VBM =6; ReHo = 2). Next, we used both VBM and ReHo data to construct the new SVM classifier, which could effectively identify patients with BPD at an accuracy of $87.5 \%$ (95\%Cl: $72.5-95.3 \%)$, sensitivity of $86.4 \%$ (95\%Cl: $64.0-96.4 \%)$, and specificity of $88.9 \%$ (95\%Cl: 63.9-98.0\%) in the test data ( $p=0.0022)$.

Conclusions: A combination of structural and functional MRI can be of added value in the construction of SVM classifiers to aid in the accurate identification of BPD in the clinic.
\end{abstract}

Keywords: Bipolar disorder, Multimodality magnetic resonance imaging, Support vector machine

\section{Background}

Bipolar disorder (BPD) is a chronic and disabling mood disorder found in up to $2.5 \%$ of the population. It is characterized by extreme fluctuations in mood, functionality, and energy, in addition to recurrent depressive and manic/

\footnotetext{
*Correspondence: cui_sysu@163.com; coolliping@163.com

${ }^{+} \mathrm{Hao} \mathrm{Li}$ and Liqian Cui contributed equally to this work.

'Department of Neurology, The First Affiliated Hospital, Sun Yat-sen University, Guangzhou, China

${ }^{3}$ Affiliated Brain Hospital of Guangzhou Medical University, Guangzhou Huiai Hospital, Guangzhou, Guangdong, China

Full list of author information is available at the end of the article
}

hypomanic episodes. Due to the early onset of the disease, high rates of self-inflicted injury and hospitalization, and the negative stigma of BPD, the disease causes significant social and economic burden $[1,2]$. It was previously reported that the risk of suicide was 20-times higher in patients with BPD than the more general population [3-6]. In addition, the clinical symptoms of BPD overlap with those of many other mood disorders, including major depressive disorder (MDD), schizophrenia, and attention deficit and hyperactivity disorder (ADHD) [7]. Due to the similar symptom profiles, BPD often goes undiagnosed or misdiagnosed for

(C) The Author(s). 2020 Open Access This article is licensed under a Creative Commons Attribution 4.0 International License, which permits use, sharing, adaptation, distribution and reproduction in any medium or format, as long as you give appropriate credit to the original author(s) and the source, provide a link to the Creative Commons licence, and indicate if changes were made. The images or other third party material in this article are included in the article's Creative Commons licence, unless indicated otherwise in a credit line to the material. If material is not included in the article's Creative Commons licence and your intended use is not permitted by statutory regulation or exceeds the permitted use, you will need to obtain permission directly from the copyright holder. To view a copy of this licence, visit http://creativecommons.org/licenses/by/4.0/ The Creative Commons Public Domain Dedication waiver (http://creativecommons.org/publicdomain/zero/1.0/) applies to the data made available in this article, unless otherwise stated in a credit line to the data. 
extended periods. In some cases, it may take up to 10 years after initially seeking treatment to be correctly diagnosed with BPD [8]. This further aggravates the effective treatment of BPD and results in increased disease burden. Hence, researchers are seeking new potential biomarkers to assist the diagnosis and therapeutic monitoring of BPD. Among the new biomarkers, neuroimaging biomarkers have shown excellent potential.

Magnetic resonance imaging (MRI) is a non-invasive neuroimaging technique used to assess the internal anatomy of the brain. In recent years, MRI has been extensively utilized in neuroimaging studies as a potential biomarker. For structural MRI, voxel-based morphometry (VBM) is one of the most common techniques used to assess focal differences in brain anatomy. The MRI scans of individuals are normalized to a standard template, and voxel-by-voxel comparisons are used to investigate localized abnormalities in gray matter density or volume [9]. For functional MRI, regional homogeneity ( $\mathrm{ReHo}$ ) is a data-driven and highly established approach to evaluate local activity in the brain while at rest. In practice, $\mathrm{ReHO}$ represents the temporal homogeneity of the regional blood oxygen level-dependent (BOLD) signal by using Kendall's coefficient of concordance (KCC), which is a number from 0 to 1 that indicates interrater agreement [10]. Fluctuations in ReHo are indicative of local abnormalities in brain activity [11]. Through both VBM and ReHo studies, BPD has been identified as a disorder with several morphological and functional brain abnormalities. However, there have been some inconsistencies between the studies. In terms of VBM studies, abnormalities have been detected extensively throughout the brain, including the frontal lobe, temporal lobe, parietal lobe, cingulate cortex, and the cerebellum. In addition, some of these findings have contradicted each other. For example, some studies shown increased gray matter volumes in the ventral prefrontal cortex (PFC) [12], inferior frontal gyrus $[13,14]$, middle temporal gyri and left temporal pole [15], cingulate gyrus [12, 16], putamen [17, 18], and cerebellum [18], while other studies found reduced gray matter volumes in the same areas [19-27]. A similar situation exists with the ReHo analysis. Some studies found increased ReHo values in the frontal lobe, cingulate cortex, and parahippocampal gyrus, while others found reduced ReHo values in the same areas [28-32]. As most of the studies mentioned above were performed at the group level, it is challenging to apply these findings to the individual identification of BPD.

Recently, machine learning approaches have introduced to address the dilemma of inconsistencies. In machine learning, the nature of the "diagnosis" is a classification problem. Among them, Support Vector Machines (SVM) have been developed from the theory of limited samples Statistical Learning Theory by Vapnik et al., which was originally designed for binary classification [33]. It is constructed based on the simplicity of structural risk minimization instead of empirical risk minimization. This enables SVM an optimal generalization ability in difficult situations [34, 35]. For these reasons, it has been widely used in the detection of mental disorders. For structural MRI, SVM has been used to accurately identify Alzheimer's disease, autism spectrum disorders MDD, obsessivecompulsive disorder, and schizophrenia [36-40]. SVM has also been used to accurately identify different mental disorders in functional MRI studies [41-43]. In terms of BPD, Redlich and colleagues previously used an SVM algorithm based on the whole-brain gray matter to distinguish between BPD and unipolar depression with an accuracy of almost 76\% [44]. In another study, SVM was used in combination with thalamic seed-based connectivity to differentiate between BPD and healthy controls (HC) with an accuracy of $61.7 \%$ [45].

However, the majority of previous studies have used single-modality MRI, either structural or functional MRI. However, a single imaging modality only provides a limited snapshot of the brain in terms of structure or function, while the combined structure-function analysis may provide a more comprehensive perspective of the brain. In recent years, multimodality MRI has been applied to SVM for the classification of schizophrenia and ASD, and the findings have verified that multimodality imaging is significantly more accurate than single modality imaging [46, 47]. Hence, in this study, we have constructed an SVM model, with VBM and ReHo measurement in gray matter volumes as features, to differentiate between patients with BPD from the HCs. We evaluated the classification capabilities of the model and identified the brain areas critical for discriminating between BPD and the HCs. To the best of our knowledge, this is the first study to distinguish between patients with BPD and HCs using an SVM classifier based on the combination of ReHo and VBM analyses.

\section{Methods \\ Participants}

Between January 2012 and December 2015, 44 patients with BPD and 36 age- and sex-matched HCs were recruited from the Affiliated Brain Hospital of Guangzhou Medical University (Guangzhou Huiai Hospital, Guangdong, China) and surrounding communities, respectively. The patients were preliminarily diagnosed as having BPD by one or more of our senior psychiatrists, based on the criteria outlined by the Diagnostic and Statistical Manual of Mental Disorders 4th Edition (DSM-IV) and the structured clinical interview for DSM-IV (SCID) for further confirmation. The HCs were also screened with SCID to ensure they had a fit mental status. All participants were from the Han population, right-handed, and had intelligence quotient scores above 75. The exclusion criteria for the BPD and HC groups were as follows: (1) comorbid Axis I or Axis II 
disease; (2) history of other psychiatric or neurological illness or severe physical illness; (3) active substance abuse or addiction; (4) unable to complete MRI session due to physical or mental limitations; (5) organic brain lesions detecting by MRI. The study was approved by institutional review boards of Guangzhou Huiai Hospital, and written informed consent was obtained from each participant or their legal guardians before the study.

\section{Collection of demographic and clinical information}

General demographic information, such as age, sex, and years of education, were collected using a pre-designed standardized form. Clinical data, including duration of illness, recurrence, medication, and clinical symptom ratings, were obtained from patients in the BPD group. The Young Mania Rating Scale (YMRS), Positive and Negative Syndrome Scale (PANSS), Hamilton Depression Rating Scale (HAMD), Hamilton Anxiety Table (HAMA), and Global Assessment Function (GAF) were applied to evaluate each subject.

\section{MRI acquisition}

MRI scans were obtained for all of the subjects by a skilled medical imaging technician on the Philips Achieva 3.0 T X-series MRI scanner with 8-channel phased array coils at Guangzhou Huiai Hospital. Conventional T1, T2, and blood oxygenation level-dependent (BOLD) images were acquired for each subject. First, 3D T1-weighted volumetric structural images were acquired using a turbo field echo 3D T1 sequence with the following parameters: repetition time $(\mathrm{TR})=8.2 \mathrm{msec}$, echo time $(\mathrm{TE})=3.8 \mathrm{msec}$, matrix size $=256 \times 256$, field of view $(\mathrm{FOV})=250 \times 250$ $\mathrm{mm}^{2}$, number of slices $=188$, slice thickness $=1 \mathrm{~mm}$, and inter-slice gap $=0 \mathrm{~mm}$.

Blood oxygenation level-dependent (BOLD) functional images were acquired using a fast field echo (FFE) echoplanar images (EPI) sequence with the following parameters: $\mathrm{TR}=2000 \mathrm{msec}, \mathrm{TE}=30 \mathrm{msec}$, flip angle $=30^{\circ}$, slice numbers $=33$, matrix size $=64 \times 64, \mathrm{FOV}=220 \times$ $220 \times 150 \mathrm{~mm}^{3}$, inter-slice gap $=0.6 \mathrm{~mm}$, and voxel size $=$ $3.44 \times 3.44 \times 4 \mathrm{~mm}$. During the fMRI scan time of $523 \mathrm{~s}$, 240 volumes were obtained. Before the scan, the subjects were instructed to "remain still, relaxed, and close eyes but not fall asleep. Try not to think actively." After the scan, the subjects were asked to confirm that they remained awake during the scanning session.

\section{Pre-processing and analysis of the structural and functional images}

For the structural images, we used the CAT 12 toolbox (http://dbm.neuro.uni-jena.de/cat/) based on the statistic parametric mapping software package (SPM12, http:// www.fil.ion.ucl.ac.uk/spm/) in the MATLAB environment (MATLAB 2018b, MathWorks, Natick, MA, USA) to accomplish the data pre-processing and analysis. First, a customized template based on our subjects was created with the segment function in CAT12 and Diffeomorphic Anatomical Registration using Exponentiated Lie (DART EL) algebra function in SPM 12 [48]. Next, the customized template was used to normalize each subject with a $1.5 \times 1.5 \times 1.5 \mathrm{~mm}^{3}$ voxel size. The normalized images were sent through the standard segmentation and modulation procedure using the default settings in CAT 12 toolbox. Lastly, an 8-mm full-width-half maximum (FWHM) Gaussian smoothing was performed to improve the signal-to-noise ratio. All images were checked for potential image defects or abnormalities.

For the functional images, we used the DPABI_v4.4 (http://www.rfmri.org/dpabi), and SPM12 toolboxes running on MATLAB 2018b were applied to pre-process functional images [49]. First, the first 10 time points were discarded to maintain a steady signal. A total of $230 \mathrm{im}$ ages for each subject were obtained and corrected for slice timing. Next, using the realign function in DPABI, we corrected the head motion, and anyone with head motions that exceeded $1.5 \mathrm{~mm}$ or rotations over $1.5^{\circ}$ were excluded. Subsequently, several spurious covariates, including the linear trend of data, white matter, cerebrospinal fluid, and the Friston-24 parameters of head motion, were removed to reduce the effects of scanning time, breathing, and heart beats [50]. Next, the images were normalized to standard Montreal Neurological Institute (MNI) space, resampling to $3 \times 3 \times 3 \mathrm{~mm}^{3}$, based on the previous customized DARTEL template. In the last step of pre-processing, temporal band-pass filtering $(0.01-0.08 \mathrm{~Hz})$ was performed to minizine the effects of low-frequency drift and high-frequency physiological noise.

We also used the DPABI toolbox to conduct an H3 ReHo analysis. First, the ReHo map was obtained by calculating Kendall's correlation coefficient (KCC) for each voxel and the 26 adjacent voxels. Next, the ReHo map was normalized by dividing the averaged $\mathrm{KCC}$ of the entire brain. Lastly, a 6-mm FWHM Gaussian smoothing was performed in the normalized ReHo map.

\section{Feature selection and construction of the Support Vector Machine (SVM)}

To obtain more sensitive features and improve the stability and efficiency of the classification in the SVM, a twosample $t$-test with multiple comparisons was used in the VBM and ReHo statistical analyses [51]. In the VBM analysis, we applied age, sex, education level, and total intracranial volume as nuisance covariates, and the t-map was corrected for multiple comparisons using the Gaussian random field (GRF) theory (voxel-level $p<0.001$, clusterlevel $p<0.05)$. The grey matter volumes of the clusters showing significant differences were obtained from each subject using the DPABI toolbox. In the ReHo analysis, 
age, sex, and education level were regarded as nuisance covariates, and the t-map was corrected using the GRF approach (voxel-level $p<0.01$, cluster-level $p<0.05$ ). The ReHo values of each subject in the clusters showing significant differences were also extracted using the DPABI toolbox. Further, between these regions showed by VBM/ Reho analyses, we used LASSO (the least absolute shrinkage selector operator) approach to identify the most informative regions and also reduce the dimensionality of the feature space to avoid over-fitting. Grey matter volumes and ReHo values in each cluster were selected as feature vectors for discrimination and inputted into the SVM to construct the final classification model.

The classification model, which is based on the support vector machine (SVM), was constructed using the LIBSVM soft package in MATLAB environment. All subjects were randomly divided into the training data and test data, where training data were used to learn the difference between groups and build the classification model, and test data were used to evaluate the classification power of the new model. In this process, leave-one-out cross-validation (LOOCV) and grid search methods were applied to ensure the stability and reliability of the model. Next, the accuracy, specificity, sensitivity, and AUC were assessed to comprehensively evaluate the classification model using a permutation test of 5000 times. Also, we compared the performance of the classification model with grey matter volumes alone and ReHo value alone, and with the combination of both grey matter volumes and ReHo.

\section{Results}

\section{Demographic and clinical information of subjects}

In this study, 44 patients diagnosed with BPD and ageand sex-matched 36 healthy controls were recruited. All subjects went through the SCID, and their images were assessed for quality control. None of the subjects were excluded for mental abnormalities, other than BPD, or other defects found in the scans. Demographic and clinical characteristics are summarized in Table 1. In terms of demographics, no significant differences were found in age or gender between the BPD and HC groups $(p>0.05)$, while the length of education was shorter in the BPD group as compared with the $\mathrm{HC}$ group. In the BPD group, the age at first onset of the disease was $21.0 \pm 5.85$, with a disease course of $2.82 \pm 1.86$ years and recurrence of $1.73 \pm 1.19$ times. In addition, significant differences in the GAF, HAMA, HAMD, and PANSS scores were found between the BPD group and $\mathrm{HC}$ group $(p<0.05)$.

\section{Feature selection}

For the VBM and Reho analysis, a total of 14 clusters showed significant differences between the groups and were extracted. 12 clusters from VBM analyses covered the bilateral inferior frontal gyrus, precentral gyrus,
Table 1 Demographic and clinical characteristics of subjects in the BPD and HC groups

\begin{tabular}{|c|c|c|c|c|}
\hline & BPD & $\mathrm{HC}$ & $t / x$ & $P$ \\
\hline Age & $23.11 \pm 5.15$ & $22.78 \pm 2.45$ & 0.3589 & 0.720 \\
\hline $\operatorname{Sex}(M / F)$ & $18 / 26$ & $22 / 14$ & 3.2323 & 0.072 \\
\hline Education (years) & $12.59 \pm 2.94$ & $15.19 \pm 1.62$ & -4.7485 & $<0.001$ \\
\hline Subtype (I / II) & $40 / 4$ & - & - & - \\
\hline \multicolumn{5}{|l|}{ Mood status } \\
\hline depressive & $4(9.1 \%)$ & - & - & - \\
\hline manic & $4(9.1 \%)$ & - & - & - \\
\hline remission & $36(81.8 \%)$ & - & - & - \\
\hline Onset-year & $21.3 \pm 5.85$ & - & - & - \\
\hline Course of disease & $2.82 \pm 1.86$ & - & - & - \\
\hline Recurrence & $1.73 \pm 1.19$ & - & - & - \\
\hline GAF & $75.95 \pm 14.36$ & $98.86 \pm 2.88$ & -9.410 & $<0.001$ \\
\hline HAMA & $3.64 \pm 3.69$ & $0.42 \pm 0.65$ & 5.170 & $<0.001$ \\
\hline HAMD & $2.91 \pm 3.85$ & $0.39 \pm 0.80$ & 3.860 & $<0.001$ \\
\hline PANSS & $41.07 \pm 11.87$ & $30.47 \pm 1.09$ & 5.335 & $<0.001$ \\
\hline YOUNG & $3.84 \pm 7.10$ & $0.03 \pm 0.17$ & 3.218 & 0.002 \\
\hline \multicolumn{5}{|l|}{ Medications } \\
\hline antipsychotics & $35(79.5 \%)$ & - & - & - \\
\hline lithium & $18(41.0 \%)$ & - & - & - \\
\hline valproate & $22(44.7 \%)$ & - & - & - \\
\hline antidepressants & $8(18.2 \%)$ & - & - & - \\
\hline
\end{tabular}

$B P D$ bipolar disorder, $H C$ healthy controls, GAF Global Assessment Function, HAMA Hamilton Anxiety Scale, HAMD Hamilton Depression Rating Scale, PANSS Positive and Negative Syndrome Scale, YMRS Young Mania Rating Scale

postcentral gyrus, middle occipital gyrus, fusiform and right middle frontal gyrus, cingulate gyrus, anterior cingulate, hippocampus, superior temporal gyrus, lingual gyrus and left limbic lobe, inferior temporal gyrus, and precuneus (GRF-corrected, voxel-level $p<0.001$, cluster-level $p<0.05$, Fig. 1). The specific grey matter volumes of these clusters were extracted and shown in Table S1 (Supplementary Materials). Two clusters from Reho analyses covered the right medial frontal gyrus, anterior cingulate, left lentiform nucleus, and putamen (GRF-corrected, voxellevel $p<0.01$, cluster-level $p<0.05$, Fig. 2). The ReHo values of the two clusters were extracted and shown in Table S2 (Supplementary Materials). And at last, 8 clusters, 6 from VBM analyses and 2 from Reho analyses, survived from LASSO selection (Table 2).

\section{SVM analysis}

Based on a combination of grey matter volume differences and ReHo values, the trained SVM classifier could correctly identify BPD with an accuracy of $87.5 \%$ (95\%CI: 72.5-95.3\%), sensitivity of $86.4 \%$ (95\%CI: $64.0-96.4 \%$ ), and specificity of $88.9 \%$ (95\%CI: 63.9-98.0\%) in the test data $(p=0.0022)$. The specific classification results from 


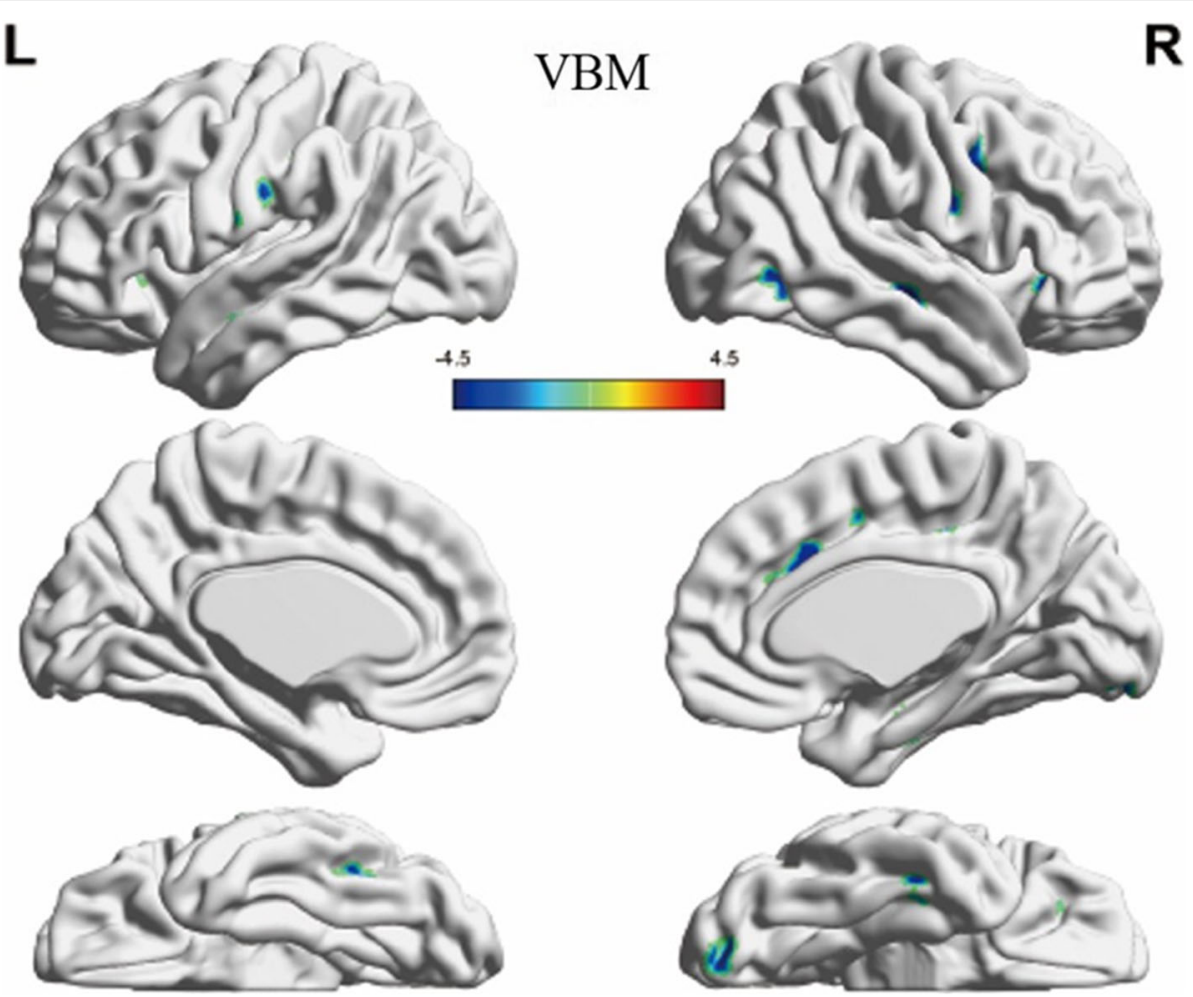

Fig. 1 Clusters showing significant differences in between the BPD and HC groups in gray matter volume

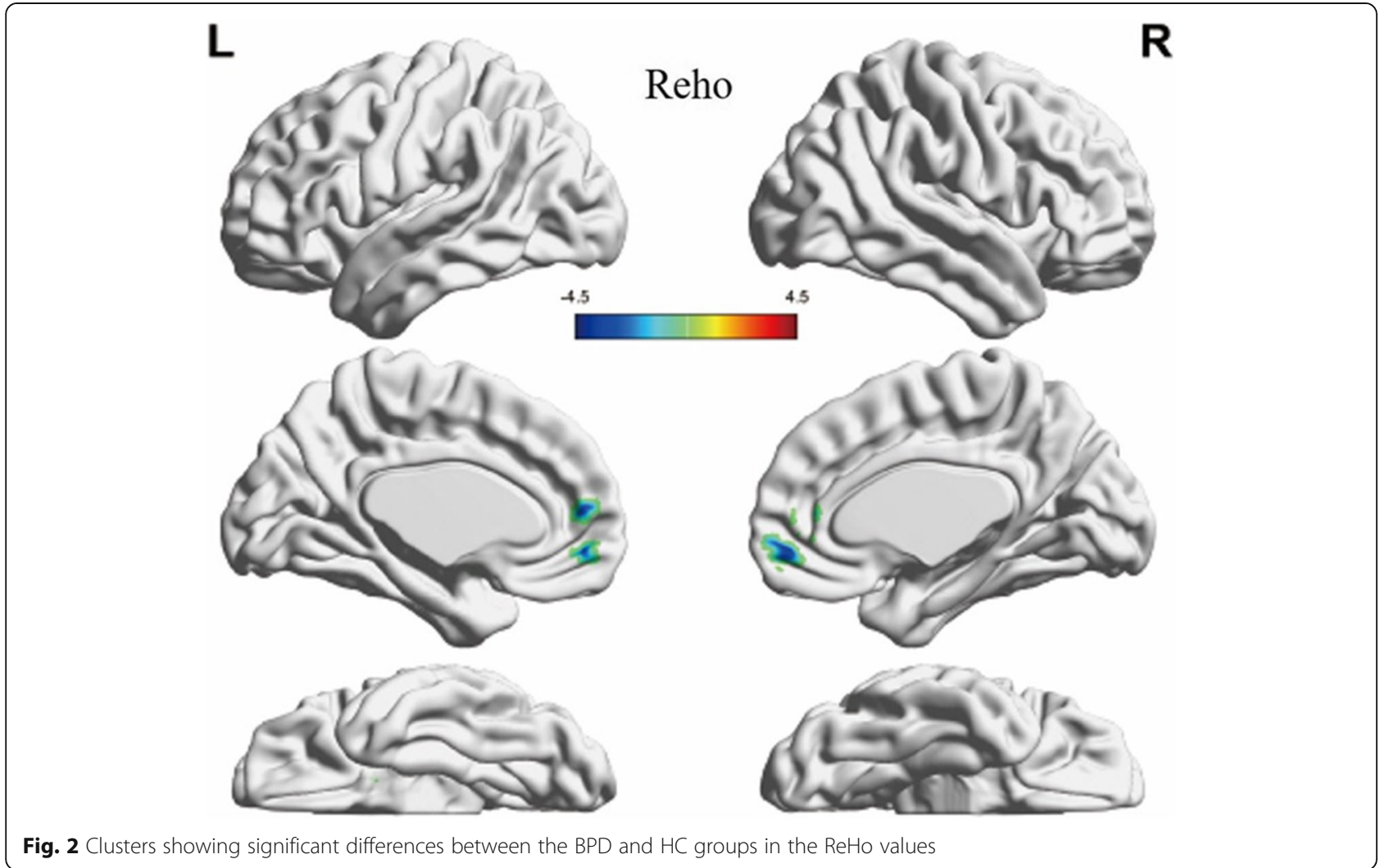

Fig. 2 Clusters showing significant differences between the BPD and HC groups in the ReHo values 
Table 2 Clusters survived from LASSO selection

\begin{tabular}{|c|c|c|c|c|c|c|}
\hline \multirow{2}{*}{$\begin{array}{l}\text { Cluster } \\
\text { number }\end{array}$} & \multirow[t]{2}{*}{ Brain regions } & \multicolumn{3}{|c|}{ Peak MNI coordinate } & \multirow{2}{*}{$\begin{array}{l}\text { Voxel } \\
\text { sizes }\end{array}$} & \multirow[t]{2}{*}{$\mathbf{T}$} \\
\hline & & $x$ & $y$ & $z$ & & \\
\hline \multicolumn{7}{|c|}{ VBM analyses } \\
\hline 1 & $\begin{array}{l}\text { Right superior temporal gyrus } \\
\text { Right hippocampus } \\
\text { Right fusiform }\end{array}$ & 45 & -20 & -14 & 891 & -5.15 \\
\hline 2 & Right lingual gyrus & 15 & -89 & -14 & 123 & -3.96 \\
\hline 3 & Left inferior frontal gyrus & -21 & 35 & 0 & 269 & -4.23 \\
\hline 4 & $\begin{array}{l}\text { Left Precentral Gyrus } \\
\text { Left Postcentral Gyrus }\end{array}$ & -54 & -15 & 28 & 851 & -4.34 \\
\hline 5 & Right Middle occipital gyrus & 35 & -66 & 29 & 381 & -4.16 \\
\hline 6 & $\begin{array}{l}\text { Left Precuneus } \\
\text { Left Middle occipital gyrus }\end{array}$ & -15 & -59 & 36 & 489 & -4.71 \\
\hline \multicolumn{7}{|c|}{ Reho analyses } \\
\hline 1 & $\begin{array}{l}\text { Left Lentiform Nucleus } \\
\text { Left Putamen }\end{array}$ & -27 & 6 & 27 & 631 & 4.34 \\
\hline 2 & $\begin{array}{l}\text { Right Medial Frontal Gyrus } \\
\text { Right Anterior Cingulate }\end{array}$ & 9 & -48 & 45 & 648 & -4.94 \\
\hline
\end{tabular}

MNI Montreal Neurological Institute

the test data are shown in Fig. 3. Based on grey matter volumes alone, the accuracy was reduced to $75 \%$ (95\%CI: $59.8-85.8 \%$ ), with a sensitivity of $72.7 \%$ (95\%CI: $49.6-$ $88.4 \%)$ and a specificity of $77.8 \%$ (95\%CI: 51.9-92.6\%), ( $p=$ 0.0122). Similarly, when based on ReHo values alone, the accuracy was reduced to $77.5 \%$ (95\%CI: $61.1-88.6 \%$ ), with increased sensitivity of $77.2 \%$ (95\%CI: $54.2-91.3 \%$ ) and specificity of $77.8 \%$ (95\%CI: $51.9-92.6 \%),(p=0.0022)$. As shown in Fig. 4, the area under the receiver operating characteristic (ROC) curves (AUC) of three SVM classifiers were 0.939 (95\%CI: 0.865-1.000), 0.795 (95\%CI: $0.652-$ 0.939 ), and 0.793 (95\%CI: 0.648-0.938), respectively.

\section{Discussion}

To the best of our knowledge, this is the first study to demonstrate the detection of individual patients with BPD using SVM classifiers based on a combination of ReHo values and grey matter volumes. We constructed the SVM classifier, which could classify BPD with an AUC of 0.949. Our findings showed that the SVM classifier based on a combination of the two performed better than the SVM classifiers based on gray matter volumes alone and ReHo values alone. This result supports our previous hypothesis that the combination of structural and functional MRI could improve the recognition of BPD using an SVM classifier. Similar findings have been detected by other research groups. Using multimodality MRI, several studies have constructed SVM classifiers for the identification of ASD, Alzheimer's Disease, and schizophrenia [46, 52, 53]. Beyond this, some researchers have combined multimodality MRI with other characteristics of these diseases, such as cerebral spinal fluid, electroencephalography, and eyetracking [54, 55]. However, markers selection requires careful consideration, as it has been shown that too much data may not improve the power of the SWM classifier in some instances [56]. The finding may be due to the process of over-fitting in machine learning, which may reduce the generalization of the classifier.

In this present study, gray matter volumes and ReHo values were chosen to build the classifiers. Both VBM and ReHo analyses reflect local abnormalities of the brain, in terms of structure and function dimensions independently. In addition, the two approaches are data-driven measurements, which are independent from preconceived assumptions and could make the findings more objective. In the VBM analyses, reduced grey matter volumes were confirmed in multiple cerebral areas, including the frontal lobe, the temporal lobe, the parietal lobe, the limbic system, and the hippocampus. This finding did not go beyond the scope of regions reported by previous literatures. Many researchers have found gray matter shrinkage across extensive frontal regions of inferior frontal gyrus (Stanfield et al., 2009; Lyoo et al., 2014; Wang et al., 2011;), middle frontal gyrus (Li et al., 2011; Brown et al., 2011) and precentral gyrus (Lyoo et al., 2014; Brown et al., 2011) [14, 21, $25,57,58]$. Inferior and middle frontal gyrus is the primary component of the prefrontal cortex and is involved in aspects of emotional regulatory processes and emotional processing. Abnormal in prefrontal cortex could result in dysfunction in regulating emotion, which is recognized as one of the most fundamental pathophysiology of BPD [59, 60]. Deficits in the gray matter of postcentral gyrus have also been reported in previous studies [61, 62]. Precentral and postcentral gyrus formed the sensorimotor network $(\mathrm{SMN})$. SMN plays a role in the process of emotion regulation, which involves the recognition and feedback of 

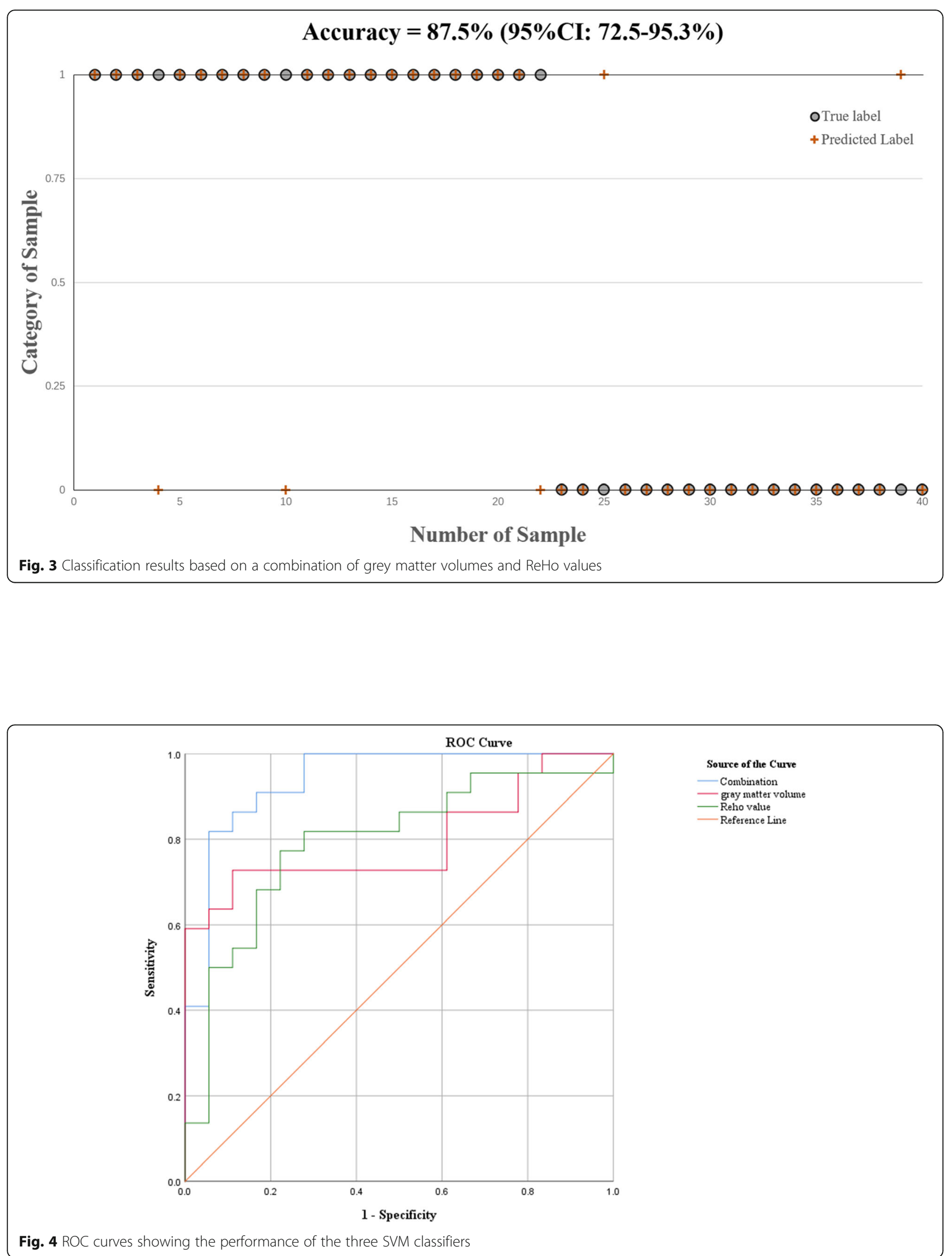

Fig. 4 ROC curves showing the performance of the three SVM classifiers 
external emotional stimuli [63, 64]. Besides, psychomotor symptoms in patients with BPD are thought to be related with sensorimotor networks $[65,66]$. As for the cingulate, anterior cingulate, limbic cortex, and hippocampus, these areas constituted the main body of limbic system. There have been many proofs that show the limbic system involving emotional processing, memory, and executive functioning, as well as its decreases of gray matter in patients with bipolar disorder [19, 23, 67-71]. Besides, we identified decreased gray matter volume in the superior temporal gyrus and inferior temporal gyrus; this finding was consistent with most previous structural MRI studies in BPD [26, 7274]. Temporal lobe is the brain region related to hearing and vision and thought to play a crucial role in emotional processing, working memory, and social cognition $[75,76]$.. Several functional MRI studies that found dysfunction of superior temporal gyrus in $\mathrm{BD}$ patients with emotional processing impairment provide further support to this deduction. However, the exact mechanism of these abnormalities is still unclear, which be related to auditory processing and involved in superior temporal gyrus [76]. The inferior temporal lobe is a part of the ventral visual pathway related to visual presentation and object recognition. A growing body of evidence suggests that visual impairment may be involved in abnormal pathophysiological processes of bipolar disorder. Researchers have found reduced background effects in visual contrast perception [77]; an autopsy report also revealed lower brain cholesterol levels and a reduction of synapses in the visual cortex [78]; fMRI study further showed dysfunction of visual cortex in patients with bipolar disorder, suggesting that patients have difficulty diverting attention from emotional faces [79]. More ever, some researchers made comparisons between different subtypes of BPD patients. They found that BPD I patients had a decreased superior temporal gyrus gray matter volume than BPD II [74]. Two previous meta-analyses also confirmed decreased gray matter volume in the superior temporal gyrus only in BPD I patients [73, 80]. It can be speculated that deficit in superior temporal gyrus is unique to BPD type I. While most of our patients were diagnosed with BPD type I. This deduction is supported by our findings. However, a large-scale study of cortical abnormalities in BPD patients failed to detect difference between BPD subtypes [81]. More extensive studies are needed to address this inconsistence. The fusiform gyrus and the lingual gyrus, as the same as the inferior temporal lobe, are also involved in visual processing. Gray matter volume reductions in these two regions have been reported in both this present research and previous studies [13, 82-85]. And the precuneus is a part of the parietal lobe, which involves a variety of complex functions, including recollection and memory, integration of information relating to the perception of the environment, episodic memory retrieval, and affective responses pain. Previous studies have reported function deficits of these processes in BPD patients [86, 87]. Our study identified diminished gray matter volume of precuneus in BPD patients; this finding is consistent with functional impairment in BPD patients and a previous metaanalysis of gray matter abnormalities on BPD patients [88]. Besides, contrast to most previous studies, we failed to find any abnormalities in insular in BPD patients. Insular has been regarded as a potential state-related region recently [80]; researchers found that deficits in insular grey matter volume were consistent in all patients with bipolar disorder, except for patients in the euthymic phase [80]. Most of the patients were in remission, which can explain our failure to find any abnormalities in insular in our study. In the ReHo analyses, decreased ReHo value of medial frontal gyrus and anterior cingulate gyrus was found in our study and also have been founded in previous studies [30,31]. While increases in ReHo were detected in the left putamen and lentiform nucleus in the current study. To our knowledge, this change was first observed in the ReHo analysis of patients with BPD. However, a series of other types of studies implicated this finding. Researchers have found increased gray matter volume and cerebral blood flow in the basal ganglia. Some fMRI studies revealed increased resting-state functional connectivity between basal ganglia and other regions, including the prefrontal cortex, precuneus, and insula $[89,90]$. Further, they found that hyperactivity of basal ganglia in bipolar depression patients was positively associated with depressive episode's duration [89]. This may be explained by biased memory caused by caudate and putamen activity in cognitive models of depression [91]. In general, most of the findings from the VBM and ReHo analyses in the present study have been found in previous studies. These areas cover a wide range of cortical regions and are thoughted to be associated with various aspects of the pathophysiological process of bipolar disorder. However, due to some opposition from other studies $[15,16,27,31$, 92], direct identification of these abnormalities as biomarkers may be preliminary. Thus, we constructed an SVM classifier with excellent performance in the identification of individual patients with BPD patients with an accuracy of approximately $87.5 \%$. Besides, using the combination of grey matter volumes and ReHo values to construct the SVM classifier, the design performed better than using either gray matter volumes or ReHo values individually. These results support our hypothesis that the combination of structural and functional MRI with an SVM classifier may aid in the detection of BPD in the clinic.

There are some limitations to the present study. It is a small and non-prospective study. Besides, we tested this SVM classifier in the one sample, instead of another independent dataset. These two defects may cause a poor generalization and low confidence of this classifier. Consider this, we performed the leave-one-out crossvalidation to improve the reliability and stability of the 
classifier. In addition, the theoretical basis of the SVM is structural risk minimization instead of empirical risk minimization, which can effectively work with some degree of error and does not require a large sample size. Another key limitation of the current study is potential drugbias. Due to ethical reasons, all patients in this study were receiving medications. Previous studies have reported increased gray matter in paracentral gyrus and superior parietal gyrus caused by lithium and reduced gray matter in visual cortex caused by antiepileptics [81]. In our study, approximately $80 \%$ of BPD patients were prescribed with mood stabilizers, and more than half of them were prescribed with valproate. Deficits in ventral visual pathway also were found in present study. Hence, it can be difficult to distinguish whether these deficits are medicationinduced or abnormalities detected in the disease itself. A further distinction may be needed using unmedicated patients with long-term follow-ups. In addition, illness duration is also an important confounder. Hibar et al. (2017) have reported that long duration of illness could lead to reduced gray matter in pericalcarine gyrus, anterior cingulate gyrus, cuneus and increased gray matter in entorhinal gyrus. However, the illness duration of BPD patients in a previous study was much longer than that in our study, more than 10 years and less than 3 years, respectively. Thus, the effect of illness duration in our study was relatively weak. Lastly, this study focused on grey matter volumes and ReHo values as discriminant features to construct the SVM classifier, yet these may not provide a comprehensive assessment of the brain. More suitable neuroimaging biomarkers, such as those to detect changes in white matter microstructures and cerebral blood flow, should be considered in future studies.

\section{Conclusions}

In this study, we have shown grey matter volumes and ReHo values, as the discriminate features, could be used to conduct SVM classifiers and recognize patients with BPD at the individual level. Compared with the singlemodality MRI, the combination of structural and functional MRI data could be of added value in the construction of SVM classifiers for the accurate detection of BPD.

\section{Supplementary information}

Supplementary information accompanies this paper at https://doi.org/10. 1186/s12888-020-02886-5.

Additional file 1: Table S1. Grey matter volume of clusters showing significant differences between groups between the BPD and HC groups. Table S2. Reho value of clusters showing significant differences between between the BPD and HC groups.

\section{Abbreviations}

BPD: Bipolar disorder; MDD: Major depressive disorder; ADHD: Attention deficit and hyperactivity disorder; HC: Healthy controls; DSM-IV: The Diagnostic and Statistical Manual of Mental Disorders 4th Edition;
SCID: Structured clinical interview for DSM-IV; YMRS: Young Mania Rating Scale; PANSS: Positive and Negative Syndrome Scale; HAMD: Hamilton Depression Rating Scale; HAMA: Hamilton Anxiety Table; GAF: Global Assessment Function; SVM: Support vector machine; AUC: Area Under Curve: ROC: Receiver operating characteristic curve; MRI: Magnetic resonance imaging; FFE: Fast field echo; EPI: Echo-planar images; TR: Repetition time; TE: Echo time; FOV: Field of view (FOV); fMRI: Functional MRI fMRl; KCC: Kendall's coefficient of concordance; BOLD: Blood oxygen leveldependent; VBM: Voxel-based morphometry; ReHo: Regional homogeneity; DARTEL: Diffeomorphic Anatomical Registration using Exponentiated Lie; FWHM: Full-width-half maximum; MNI: Montreal Neurological Institute space; GRF: Gaussian random field; LASSO: The least absolute shrinkage selector operator; SMN: Sensorimotor network; PFC: Prefrontal cortex

\section{Acknowledgements}

Not applicable.

\section{Authors' contributions}

Hao Li and Liqian Cui contributed equally to this work; Conception and design of study: Hao Li, Liqian Cui; Acquisition of data: Yizhi Zhang, Yueheng Liu, Wenhao Deng; Analysis and/or interpretation of data: Hao Li, Wenjin Zhou; Drafting the manuscript: Hao Li; Revising the manuscript: Liqian Cui, Liping Cao, Yueheng Liu; Approval of the version of the manuscript to be published: Hao Li, Liqian Cui, Liping Cao, Yizhi Zhang, Yueheng Liu, Wenhao Deng, and Wenjin Zhou. The author(s) read and approved the final manuscript.

\section{Funding}

This study was supported by grants from the Southern China International Cooperation Base for Early Intervention and Functional Rehabilitation of Neurological Diseases (2015B050501003), Guangdong Provincial Engineering Center For Major Neurological Disease Treatment, Guangdong Provincial Translational Medicine Innovation Platform for Diagnosis and Treatment of Major Neurological Disease, Guangdong Provincial Clinical Research Center for Neurological Diseases. The funding body was not involved in the design of the study, data collection and analysis, interpretation of data, and writing the manuscript

\section{Availability of data and materials}

The data used to support the findings of this study are available from the corresponding author upon request.

Ethics approval and consent to participate

The study was approved by institutional review boards of Guangzhou Huiai Hospital, and written informed consent was obtained from each participant or their legal guardians before the study.

Consent for publication

Not applicable.

\section{Competing interests}

The authors declare no potential conflicts of interest.

\section{Author details}

'Department of Neurology, The First Affiliated Hospital, Sun Yat-sen University, Guangzhou, China. ${ }^{2}$ Guangdong Provincial Key Laboratory of Diagnosis and Treatment of Major Neurological Diseases, National Key Clinical Department and Key Discipline of Neurology, No.58 Zhongshan Road 2, Guangzhou 510080, China. ${ }^{3}$ Affiliated Brain Hospital of Guangzhou Medical University, Guangzhou Huiai Hospital, Guangzhou, Guangdong, China.

${ }^{4}$ Department of Psychiatry, The Second Xiangya Hospital, Central South University, Changsha, Hunan, China. ${ }^{5}$ Chinese National Clinical Research Center on Mental Disorders (Xiangya), Changsha, Hunan, China.

Received: 14 February 2020 Accepted: 21 September 2020

Published online: 06 October 2020

References

1. Alonso J, Petukhova M, Vilagut $G$, Chatterji S, Heeringa S, Üstün TB, Alhamzawi AO, Viana MC, Angermeyer M, Bromet $E$, Bruffaerts $R$, de Girolamo G, Florescu S, Gureje O, Haro JM, Hinkov H, Hu CY, Karam EG, 
Kovess V, Levinson D, Medina-Mora ME, Nakamura Y, Ormel J, Posada-Villa J, Sagar R, Scott KM, Tsang A, Williams DR, Kessler RC. Days out of role due to common physical and mental conditions: results from the WHO World Mental Health surveys. Mol Psychiatry. 2011;16:1234-46.

2. Simon GE. Social and economic burden of mood disorders. Biol Psychiatry. 2003;54:208-15.

3. Pompili M, Gonda X, Serafini G, Innamorati M, Sher L, Amore M, Rihmer Z, Girardi P. Epidemiology of suicide in bipolar disorders: a systematic review of the literature. Bipolar Disord. 2013;15:457-90.

4. Plans L, Barrot C, Nieto E, Rios J, Schulze TG, Papiol S, Mitjans M, Vieta E, Benabarre A. Association between completed suicide and bipolar disorder: A systematic review of the literature. J Affect Disord. 2019;242:111-22.

5. Malhi GS, Outhred T, Das P, Morris G, Hamilton A, Mannie Z. Modeling suicide in bipolar disorders. Bipolar Disord. 2018;20:334-48.

6. Hansson C, Joas E, Pålsson E, Hawton K, Runeson B, Landén M. Risk factors for suicide in bipolar disorder: a cohort study of 12850 patients. Acta Psychiatr Scand. 2018;138:456-63.

7. Pendergast LL, Youngstrom EA, Merkitch KG, Moore KA, Black CL, Abramson LY, Alloy LB. Differentiating bipolar disorder from unipolar depression and ADHD: the utility of the general behavior inventory. Psychol Assess. 2014;26:195-206.

8. Hirschfeld RM, Lewis L, Vornik LA. Perceptions and impact of bipolar disorder: how far have we really come? Results of the national depressive and manic-depressive association 2000 survey of individuals with bipolar disorder. J Clin Psychiatry. 2003;64:161-74.

9. Ashburner J, Friston KJ. Voxel-based morphometry--the methods. Neuroimage. 2000;11:805-21.

10. Zang Y, Jiang T, Lu Y, He Y, Tian L. Regional homogeneity approach to fMRI data analysis. Neuroimage. 2004;22:394-400.

11. Wu QZ, Li DM, Kuang WH, Zhang TJ, Lui S, Huang XQ, Chan RC, Kemp GJ, Gong QY. Abnormal regional spontaneous neural activity in treatment-refractory depression revealed by resting-state fMRI. Hum Brain Mapp. 2011;32:1290-9.

12. Adler CM, Levine AD, DelBello MP, Strakowski SM. Changes in gray matter volume in patients with bipolar disorder. Biol Psychiatry. 2005;58:151-7.

13. Sarıççek A, Yalın N, Hıdıroğlu C, Çavuşoğlu B, Taş C, Ceylan D, Zorlu N, Ada E, Tunca Z, Özerdem A. Neuroanatomical correlates of genetic risk for bipolar disorder: a voxel-based morphometry study in bipolar type I patients and healthy first degree relatives. J Affect Disord. 2015;186:110-8.

14. Stanfield AC, Moorhead TW, Job DE, McKirdy J, Sussmann JE, Hall J, Giles S, Johnstone EC, Lawrie SM, McIntosh AM. Structural abnormalities of ventrolateral and orbitofrontal cortex in patients with familial bipolar disorder. Bipolar Disord. 2009;11:135-44.

15. Haldane M, Cunningham G, Androutsos C, Frangou S. Structural brain correlates of response inhibition in bipolar disorder I. J Psychopharmacol (Oxford). 2008;22:138-43.

16. Adler CM, DelBello MP, Jarvis K, Levine A, Adams J, Strakowski SM. Voxelbased study of structural changes in first-episode patients with bipolar disorder. Biol Psychiatry. 2007;61(6):776-81.

17. Chen Z, Cui L, Li M, Jiang L, Deng W, Ma X, Wang Q, Huang C, Wang Y, Collier DA, Gong Q, Li T. Voxel based morphometric and diffusion tensor imaging analysis in male bipolar patients with first-episode mania. Prog Neuropsychopharmacol Biol Psychiatry. 2012;36:231-8.

18. Cui L, Li M, Deng W, Guo W, Ma X, Huang C, Jiang L, Wang Y, Collier DA, Gong Q, Li T. Overlapping clusters of gray matter deficits in paranoid schizophrenia and psychotic bipolar mania with family history. Neurosci Lett. 2011;489:94-8.

19. Almeida JR, Akkal D, Hassel S, Travis MJ, Banihashemi L, Kerr N, Kupfer DJ, Phillips ML. Reduced gray matter volume in ventral prefrontal cortex but not amygdala in bipolar disorder: significant effects of gender and trait anxiety. Psychiatry Res. 2009;171:54-68.

20. Cai Y, Liu J, Zhang L, Liao M, Zhang Y, Wang L, Peng H, He Z, Li Z, Li W, Lu S, Ding Y, Li L. Grey matter volume abnormalities in patients with bipolar I depressive disorder and unipolar depressive disorder: a voxel-based morphometry study. Neurosci Bull. 2015;31:4-12.

21. Brown GG, Lee JS, Strigo IA, Caligiuri MP, Meloy MJ, Lohr J. Voxel-based morphometry of patients with schizophrenia or bipolar I disorder: a matched control study. Psychiatry Res. 2011;194:149-56.

22. Tost H, Ruf M, Schmäl C, Schulze TG, Knorr C, Vollmert C, Bösshenz K, Ende G, Meyer-Lindenberg A, Henn FA, Rietschel M. Prefrontal-temporal gray matter deficits in bipolar disorder patients with persecutory delusions. Affect Disord. 2010;120:54-61.
23. Yatham LN, Lyoo IK, Liddle P, Renshaw PF, Wan D, Lam RW, Hwang J. A magnetic resonance imaging study of mood stabilizer- and neurolepticnaïve first-episode mania. Bipolar Disord. 2007:9:693-7.

24. Haller S, Xekardaki A, Delaloye C, Canuto A, Lövblad KO, Gold G, Giannakopoulos P. Combined analysis of grey matter voxel-based morphometry and white matter tract-based spatial statistics in late-life bipolar disorder. J Psychiatry Neurosci. 2011;36:391-401.

25. Wang F, Kalmar JH, Womer FY, Edmiston EE, Chepenik LG, Chen R, Spencer L, Blumberg HP. Olfactocentric paralimbic cortex morphology in adolescents with bipolar disorder. Brain. 2011;134:2005-12.

26. Narita K, Suda M, Takei Y, Aoyama Y, Majima T, Kameyama M, Kosaka H, Amanuma M, Fukuda M, Mikuni M. Volume reduction of ventromedial prefrontal cortex in bipolar II patients with rapid cycling: a voxel-based morphometric study. Prog Neuro Psychopharmacol Biol Psychiatry. 2011;35: 439-45.

27. Frangou S. Brain structural and functional correlates of resilience to Bipolar Disorder. Front Hum Neurosci. 2011;5:184.

28. Yao X, Yin Z, Liu F, Wei S, Zhou Y, Jiang X, Wei Y, Xu K, Wang F, Tang Y. Shared and distinct regional homogeneity changes in bipolar and unipolar depression. Neurosci Lett. 2018;673:28-32.

29. Liu CH, Ma X, Li F, Wang YJ, Tie CL, Li SF, Chen TL, Fan TT, Zhang Y, Dong J, Yao L, Wu X, Wang CY. Regional homogeneity within the default mode network in bipolar depression: a resting-state functional magnetic resonance imaging study. PLoS One. 2012;7:e48181.

30. Liang MJ, Zhou Q, Yang KR, Yang XL, Fang J, Chen WL, Huang Z. Identify changes of brain regional homogeneity in bipolar disorder and unipolar depression using resting-state FMRI. PLoS One. 2013;8:e79999.

31. Gao W, Jiao Q, Lu S, Zhong Y, Qi R, Lu D, Xiao Q, Yang F, Lu G, Su L. Alterations of regional homogeneity in pediatric bipolar depression: a resting-state fMRI study. BMC Psychiatry. 2014;14:222.

32. Xiao Q, Zhong Y, Lu D, Gao W, Jiao Q, Lu G, Su L. Altered regional homogeneity in pediatric bipolar disorder during manic state: a restingstate fMRI study. PLoS One. 2013;8:e57978.

33. Vapnik $V$. The nature of statistical learning theory. New York: Springer Verlag;1995

34. Hearst MA, Dumais ST, Osuna E, Platt J, Scholkopf B. Support vector machines. IEEE Intell Syst Appl. 1998;13:18-28.

35. Belousov Al, Verzakov SA, Von Frese J. A flexible classification approach with optimal generalisation performance: support vector machines. Chemometr Intell Lab. 2002;64:15-25.

36. Oliveira PP, Nitrini R, Busatto G, Buchpiguel C, Sato JR, Amaro E. Use of SVM methods with surface-based cortical and volumetric subcortical measurements to detect Alzheimer's disease. J Alzheimers Dis. 2010;19: 1263-72.

37. Ecker C, Marquand A, Mourão-Miranda J, Johnston P, Daly EM, Brammer MJ, Maltezos S, Murphy CM, Robertson D, Williams SC, Murphy DG. Describing the brain in autism in five dimensions--magnetic resonance imagingassisted diagnosis of autism spectrum disorder using a multiparameter classification approach. J Neurosci. 2010;30:10612-23.

38. Mwangi B, Ebmeier KP, Matthews K, Steele JD. Multi-Centre diagnostic classification of individual structural neuroimaging scans from patients with major depressive disorder. Brain. 2012;135:1508-21.

39. Zhou $C$, Cheng Y, Ping L, Xu J, Shen $Z$, Jiang L, Shi L, Yang S, Lu Y, Xu X. Support vector machine classification of obsessive-compulsive disorder based on whole-brain Volumetry and diffusion tensor imaging. Front Psychiatry. 2018;9:524.

40. Xiao Y, Yan Z, Zhao Y, Tao B, Sun H, Li F, Yao L, Zhang W, Chandan S, Liu J, et al. Support vector machine-based classification of first episode drugnaïve schizophrenia patients and healthy controls using structural MRI. Schizophr Res. 2019;214:11-17.

41. Anderson JS, Nielsen JA, Froehlich AL, DuBray MB, Druzgal TJ, Cariello AN, Cooperrider JR, Zielinski BA, Ravichandran C, Fletcher PT, Alexander AL, Bigler ED, Lange N, Lainhart JE. Functional connectivity magnetic resonance imaging classification of autism. Brain. 2011;134:3742-54.

42. Zeng LL, Shen H, Liu L, Wang L, Li B, Fang P, Zhou Z, Li Y, Hu D. Identifying major depression using whole-brain functional connectivity: a multivariate pattern analysis. Brain. 2012;135:1498-507.

43. Wang S, Zhang Y, Lv L, Wu R, Fan X, Zhao J, Guo W. Abnormal regional homogeneity as a potential imaging biomarker for adolescent-onset schizophrenia: a resting-state fMRI study and support vector machine analysis. Schizophr Res. 2018;192:179-84. 
44. Redlich R, Almeida JJ, Grotegerd D, Opel N, Kugel H, Heindel W, Arolt V, Phillips ML, Dannlowski U. Brain morphometric biomarkers distinguishing unipolar and bipolar depression. A voxel-based morphometry-pattern classification approach. JAMA Psychiat. 2014;71:1222-30.

45. Anticevic A, Cole MW, Repovs G, Murray JD, Brumbaugh MS, Winkler AM, Savic A Krystal JH, Pearlson GD, Glahn DC. Characterizing thalamo-cortical disturbances in schizophrenia and bipolar illness. Cereb Cortex. 2014;24:3116-30.

46. Sen B, Borle NC, Greiner R, Brown M. A general prediction model for the detection of $\mathrm{ADHD}$ and autism using structural and functional MRI. PLoS One. 2018;13:e0194856.

47. Cabral C, Kambeitz-Ilankovic L, Kambeitz J, Calhoun VD, Dwyer DB, von Saldern S, Urquijo MF, Falkai P, Koutsouleris N. Classifying schizophrenia using multimodal multivariate pattern recognition analysis: evaluating the impact of individual clinical profiles on the Neurodiagnostic performance. Schizophr Bull. 2016;42(Suppl 1):S110-7.

48. Shen S, Sterr A. Is DARTEL-based voxel-based morphometry affected by width of smoothing kernel and group size? A study using simulated atrophy. J Magn Reson Imaging. 2013;37:1468-75.

49. Yan CG, Wang XD, Zuo XN, Zang YF. DPABI: Data Processing \& Analysis for (resting-state) brain imaging. Neuroinformatics. 2016;14:339-51.

50. Friston KJ, Williams S, Howard R, Frackowiak RS, Turner R. Movement-related effects in fMRI time-series. Magn Reson Med. 1996;35:346-55.

51. Wang S, Wang G, Lv H, Wu R, Zhao J, Guo W. Abnormal regional homogeneity as potential imaging biomarker for psychosis risk syndrome: a resting-state fMRI study and support vector machine analysis. Sci Rep. 2016; 6:27619.

52. Park JE, Park B, Kim SJ, Kim HS, Choi CG, Jung SC, Oh JY, Lee JH, Roh JH, Shim WH. Improved Diagnostic Accuracy of Alzheimer's Disease by Combining Regional Cortical Thickness and Default Mode Network Functional Connectivity: Validated in the Alzheimer's Disease Neuroimaging Initiative Set. Korean J Radiol. 2017;18(6):983-91.

53. Lee J, Chon MW, Kim H, Rathi Y, Bouix S, Shenton ME, Kubicki M. Diagnostic value of structural and diffusion imaging measures in schizophrenia. Neuroimage Clin. 2018;18:467-74.

54. Shaffer JL, Petrella JR, Sheldon FC, Choudhury KR, Calhoun VD, Coleman RE, Doraiswamy PM. Predicting cognitive decline in subjects at risk for Alzheimer disease by using combined cerebrospinal fluid, MR imaging, and PET biomarkers. Radiology. 2013;266:583-91.

55. Ding X, Yue X, Zheng R, Bi C, Li D, Yao G. Classifying major depression patients and healthy controls using EEG, eye tracking and galvanic skin response data. J Affect Disord. 2019;251:156-61.

56. Nguyen $\mathrm{MH}$, De la Torre F. Optimal feature selection for support vector machines. Pattern Recogn. 2010;43:584-91.

57. Lyoo IK, Kim MJ, Stoll AL, Demopulos CM, Parow AM, Dager SR, Friedman SD, Dunner DL, Renshaw PF. Frontal lobe gray matter density decreases in bipolar I disorder. Biol Psychiatry. 2004;55:648-51.

58. Li M, Cui L, Deng W, Ma X, Huang C, Jiang L, Wang Y, Collier DA, Gong Q, Li T. Voxel-based morphometric analysis on the volume of gray matter in bipolar I disorder. Psychiatry Res. 2011;191(2):92-97.

59. Chen $\mathrm{CH}$, Suckling J, Lennox BR, Ooi C, Bullmore ET. A quantitative metaanalysis of fMRI studies in bipolar disorder. Bipolar Disord. 2011;13(1):1-15.

60. Strakowski SM, Adler CM, Almeida J, Altshuler LL, Blumberg HP, Chang KD, DelBello MP, Frangou S, McIntosh A, Phillips ML, et al. The functional neuroanatomy of bipolar disorder: a consensus model. Bipolar Disord. 2012; 14(4):313-25.

61. Frazier JA, Breeze JL, Makris N, Giuliano AS, Herbert MR, Seidman L, Biederman J, Hodge SM, Dieterich ME, Gerstein ED, et al. Cortical gray matter differences identified by structural magnetic resonance imaging in pediatric bipolar disorder. Bipolar Disord. 2005;7(6):555-69.

62. Nugent AC, Milham MP, Bain EE, Mah L, Cannon DM, Marrett S, Zarate CA, Pine DS, Price JL, Drevets WC. Cortical abnormalities in bipolar disorder investigated with MRI and voxel-based morphometry. Neuroimage. 2006; 30(2):485-97.

63. Adolphs R, Damasio H, Tranel D, Cooper G, Damasio AR. A role for somatosensory cortices in the visual recognition of emotion as revealed by three-dimensional lesion mapping. J Neurosci. 2000;20:2683-90.

64. Adolphs R. Cognitive neuroscience of human social behaviour. Nat Rev Neurosci. 2003;4(3):165-78.

65. Cassano GB, Rucci P, Benvenuti A, Miniati M, Calugi S, Maggi L, Pini S, Kupfer DJ, Maj M, Fagiolini A, et al. The role of psychomotor activation in discriminating unipolar from bipolar disorders: a classification-tree analysis. J Clin Psychiatry. 2012;73(1):22-28.

66. Angst J, Gamma A, Bowden CL, Azorin JM, Perugi G, Vieta E, Young AH. Evidence-based definitions of bipolar-I and bipolar-II disorders among 5,635 patients with major depressive episodes in the Bridge Study: validity and comorbidity. Eur Arch Psychiatry Clin Neurosci. 2013;263(8):663-73.

67. Gasquoine PG. Localization of function in anterior cingulate cortex: from psychosurgery to functional neuroimaging. Neurosci Biobehav Rev. 2013;37: 340-8.

68. Houenou J, Frommberger J, Carde S, Glasbrenner M, Diener C, Leboyer M, Wessa M. Neuroimaging-based markers of bipolar disorder: evidence from two meta-analyses. J Affect Disord. 2011;132(3):344-55.

69. Ellison-Wright I, Bullmore E. Anatomy of bipolar disorder and schizophrenia: a meta-analysis. Schizophr Res. 2010;117(1):1-12.

70. Hajek T, Kopecek M, Höschl C, Alda M. Smaller hippocampal volumes in patients with bipolar disorder are masked by exposure to lithium: a metaanalysis. J Psychiatry Neurosci. 2012;37(5):333-43.

71. Hartberg CB, Jørgensen KN, Haukvik UK, Westlye LT, Melle I, Andreassen OA, Agartz I. Lithium treatment and hippocampal subfields and amygdala volumes in bipolar disorder. Bipolar Disord. 2015;17(5):496-506.

72. Wise T, Radua J, Via E, Cardoner N, Abe O, Adams TM, Amico F, Cheng Y, Cole JH, de Azevedo MPC, et al. Common and distinct patterns of greymatter volume alteration in major depression and bipolar disorder: evidence from voxel-based meta-analysis. Mol Psychiatry. 2017;22(10):145563.

73. Lu X, Zhong Y, Ma Z, Wu Y, Fox PT, Zhang N, Wang C. Structural imaging biomarkers for bipolar disorder: meta-analyses of whole-brain voxel-based morphometry studies. Depress Anxiety. 2019;36:353-64.

74. Ha TH, Ha K, Kim JH, Choi JE. Regional brain gray matter abnormalities in patients with bipolar II disorder: a comparison study with bipolar I patients and healthy controls. Neurosci Lett. 2009;456:44-8.

75. Allison T, Puce A, McCarthy G. Social perception from visual cues: role of the STS region. Trends Cogn Sci. 2000;4:267-78.

76. Gallagher HL, Frith CD. Functional imaging of 'theory of mind'. Trends Cogn Sci. 2003;7:77-83.

77. M. Schallmo, S.R. Sponheim, C.A. Olman, Reduced contextual effects on visual contrast perception in schizophrenia and bipolar affective disorder., 2015, pp. 3527-3537.

78. Beasley CL, Honer WG, Bergmann K, Falkai P, Lütjohann D, Bayer TA. Reductions in cholesterol and synaptic markers in association cortex in mood disorders. Bipolar Disord. 2005;7(5):449-55.

79. A.S. Garrett, A.L. Reiss, M.E. Howe, R.G. Kelley, M.K. Singh, N.E. Adleman, A. Karchemskiy, K.D. Chang, Abnormal amygdala and prefrontal cortex activation to facial expressions in pediatric bipolar disorder., 2012, pp. 821831.

80. Wang X, Luo Q, Tian F, Cheng B, Qiu L, Wang S, He M, Wang H, Duan M, Jia Z. Brain grey-matter volume alteration in adult patients with bipolar disorder under different conditions: a voxel-based meta-analysis. J Psychiatry Neurosci. 2019;44(2):89-101.

81. D.P. Hibar, L.T. Westlye, N.T. Doan, N. Jahanshad, J.W. Cheung, C.R.K. Ching, A. Versace, A.C. Bilderbeck, A. Uhlmann, B. Mwangi, B. Krämer, B. Overs, C.B. Hartberg, C. Abé, D. Dima, D. Grotegerd, E. Sprooten, E. Bøen, E. Jimenez, F. M. Howells, G. Delvecchio, H. Temmingh, J. Starke, J.R.C. Almeida, J.M. Goikolea, J. Houenou, L.M. Beard, L. Rauer, L. Abramovic, M. Bonnin, M.F. Ponteduro, M. Keil, M.M. Rive, N. Yao, N. Yalin, P. Najt, P.G. Rosa, R. Redlich, S. Trost, S. Hagenaars, S.C. Fears, S. Alonso-Lana, T.G.M. van Erp, T. Nickson, T.M. Chaim-Avancini, T.B. Meier, T. Elvsåshagen, U.K. Haukvik, W.H. Lee, A.H. Schene, A.J. Lloyd, A.H. Young, A. Nugent, A.M. Dale, A. Pfennig, A.M. Mclntosh, B. Lafer, B.T. Baune, C.J. Ekman, C.A. Zarate, C.E. Bearden, C. Henry, C. Simhand, C. McDonald, C. Bourne, D.J. Stein, D.H. Wolf, D.M. Cannon, D.C. Glahn, D.J. Veltman, E. Pomarol-Clotet, E. Vieta, E.J. Canales-Rodriguez, F.G. Nery, F.L.S. Duran, G.F. Busatto, G. Roberts, G.D. Pearlson, G.M. Goodwin, H. Kugel, H.C. Whalley, H.G. Ruhe, J.C. Soares, J.M. Fullerton, J.K. Rybakowski, J. Savitz, K.T. Chaim, M. Fatjó-Vilas, M.G. Soeiro-de-Souza, M.P. Boks, M.V. Zanetti, M.C.G. Otaduy, M.S. Schaufelberger, M. Alda, M. Ingvar, M.L. Phillips, M.J. Kempton, M. Bauer, M. Landén, N.S. Lawrence, N.E.M. van Haren, N.R. Horn, N.B. Freimer, O. Gruber, P.R. Schofield, P.B. Mitchell, R.S. Kahn, R. Lenroot, R. Machado-Vieira, R.A. Ophoff, S. Sarró, S. Frangou, T.D. Satterthwaite, T. Hajek, U. Dannlowski, U.F. Malt, V. Arolt, W.F. Gattaz, W.C. Drevets, X. Caseras, I. Agartz, P.M. Thompson, O.A. Andreassen, Cortical 
abnormalities in bipolar disorder: an MRI analysis of 6503 individuals from the ENIGMA Bipolar Disorder Working Group., 2018, pp. 932-942.

82. Y. Bai, M. Chen, J. Hsu, K. Huang, P. Tu, W. Chang, T. Su, C.T. Li, W. Lin, S. Tsai, A comparison study of metabolic profiles, immunity, and brain gray matter volumes between patients with bipolar disorder and depressive disorder., 2020, pp. 42.

83. S. Poletti, V. Aggio, T.A. Hoogenboezem, O. Ambrée, H. de Wit, A.J.M. Wijkhuijs, C. Locatelli, C. Colombo, V. Arolt, H.A. Drexhage, F. Benedetti, Brain-derived Neurotrophic Factor (BDNF) and gray matter volume in bipolar disorder., 2017, pp. 33-37.

84. Ekman CJ, Petrovic P, Johansson AG, Sellgren C, Ingvar M, Landén M. A History of Psychosis in Bipolar Disorder is Associated With Gray Matter Volume Reduction. Schizophr Bull. 2017;43(1):99-107.

85. V. Oertel-Knöchel, B. Reinke, R. Feddern, A. Knake, C. Knöchel, D. Prvulovic, J. Pantel, D.E.J. Linden, Episodic memory impairments in bipolar disorder are associated with functional and structural brain changes., 2014, pp. 830-845.

86. Bora $\mathrm{E}$, Yucel M, Pantelis C. Cognitive endophenotypes of bipolar disorder: a meta-analysis of neuropsychological deficits in euthymic patients and their first-degree relatives. J Affect Disord. 2009;1 13(1-2):1-20.

87. L.S. Schenkel, M. Marlow-O'Connor, M. Moss, J.A. Sweeney, M.N. Pavuluri, Theory of mind and social inference in children and adolescents with bipolar disorder., 2008, pp. 791-800.

88. M.J. McCarthy, S. Liang, A.D. Spadoni, J.R. Kelsoe, A.N. Simmons, Whole brain expression of bipolar disorder associated genes: structural and genetic analyses., 2014, pp. e100204.

89. He Z, \Sheng W, Lu F, Long Z, Han S, Pang Y, Chen Y. Luo W, Yu Y, Nan X, Cui Q, Chen H. Altered resting-state cerebral blood flow and functional connectivity of striatum in bipolar disorder and major depressive disorder. 2019, pp. 177-85.

90. Chen P, Chen F, Chen G, Zhong S, Gong J, Zhong H, Ye T, Tang G, Wang J, Luo $Z$, et al. Inflammation is associated with decreased functional connectivity of insula in unmedicated bipolar disorder. Brain Behav Immun. 2020.

91. S.G. Disner, C.G. Beevers, E.A.P. Haigh, A.T. Beck, Neural mechanisms of the cognitive model of depression., 2011, pp. 467-477.

92. Q. Xiao, D. Cui, Q. Jiao, Y. Zhong, W. Cao, G. Lu, L. Su, Altered regional homogeneity in pediatric bipolar disorder during manic and euthymic state: a resting-state fMRI study., 2019, pp. 1789-1798.

\section{Publisher's Note}

Springer Nature remains neutral with regard to jurisdictional claims in published maps and institutional affiliations.

Ready to submit your research? Choose BMC and benefit from:

- fast, convenient online submission

- thorough peer review by experienced researchers in your field

- rapid publication on acceptance

- support for research data, including large and complex data types

- gold Open Access which fosters wider collaboration and increased citations

- maximum visibility for your research: over $100 \mathrm{M}$ website views per year

At BMC, research is always in progress.

Learn more biomedcentral.com/submissions 